\title{
Roles of low-density lipoprotein receptor-related protein 1 in tumors
}

\author{
Peipei Xing 1,2, Zhichao Liao 1,2, Zhiwu Ren 1,2, Jun Zhao 1,2, Fengju Song ${ }^{2,3}$, Guowen Wang ${ }^{1,2}$, Kexin Chen ${ }^{2,3}$ \\ and Jilong Yang ${ }^{1,2^{*}}$
}

\begin{abstract}
Low-density lipoprotein receptor-related protein 1 (LRP1, also known as CD91), a multifunctional endocytic and cell signaling receptor, is widely expressed on the surface of multiple cell types such as hepatocytes, fibroblasts, neurons, astrocytes, macrophages, smooth muscle cells, and malignant cells. Emerging in vitro and in vivo evidence demonstrates that LRP1 is critically involved in many processes that drive tumorigenesis and tumor progression. For example, LRP1 not only promotes tumor cell migration and invasion by regulating matrix metalloproteinase (MMP)-2 and MMP-9 expression and functions but also inhibits cell apoptosis by regulating the insulin receptor, the serine/ threonine protein kinase signaling pathway, and the expression of Caspase-3. LRP1-mediated phosphorylation of the extracellular signal-regulated kinase pathway and c-jun $\mathrm{N}$-terminal kinase are also involved in tumor cell proliferation and invasion. In addition, LRP1 has been shown to be down-regulated by microRNA-205 and methylation of LRP1 CpG islands. Furthermore, a novel fusion gene, LRP1-SNRNP25, promotes osteosarcoma cell invasion and migration. Only by understanding the mechanisms of these effects can we develop novel diagnostic and therapeutic strategies for cancers mediated by LRP1.
\end{abstract}

Keywords: Low-density lipoprotein receptor-related protein 1, Tumorigenesis, Invasion, migration, Proliferation, apoptosis, Signaling pathway, MicroRNA, Fusion gene

\section{Background}

Low-density lipoprotein receptor-related protein 1 (LRP1, also known as CD91), a large endocytic receptor initially described by Herz et al. [1] in 1988, belongs to the low-density lipoprotein receptor (LDLR) superfamily. LRP1 is the most multifunctional member of this superfamily $[1,2]$. Many translational studies have shown that LRP1 is involved in two major physiological processes: endocytosis and regulation of signaling pathways [3-5]. LRP1 mediates the endocytosis of a diverse set of extracellular ligands that play important roles in tumor progression [6]. In addition, LRP1 can initiate and regulate diverse signaling pathways, including mitogenactivated protein kinase (MAPK), insulin receptor (IR), serine/threonine protein kinase (AKT), extracellular

\footnotetext{
*Correspondence: yangjilong@tjmuch.com

${ }^{1}$ Department of Bone and Soft Tissue Tumor, Tianjin Medical University

Cancer Institute and Hospital, Tianjin 30060, P. R. China

Full list of author information is available at the end of the article
}

signal-regulated kinase (ERK), and c-jun N-terminal kinase (JNK) pathways $[7,8]$. Notably, these two physiological processes play an important role in multiple biological activities, including regulation of cell growth and differentiation, modulation of cytoskeletal organization angiogenesis, cell migration, and regulation of protease degradation for tissue invasion. Hence, in this review, we focus on recent translational studies that describe the roles of LRP1 in cancer cell proliferation, apoptosis, migration, invasion, and angiogenesis.

\section{From structure to function}

The LDLR superfamily includes many members, such as LRP1, megalin (LRP2), LRP3, LRP4, LRP5, LRP6, very low-density lipoprotein receptor (VLDLR), and apolipoprotein E (apoE) receptor type 2 (apoER2, also known as LRP8) [9]. These proteins share five structural domains: (1) ligand-binding type cysteine-rich repeats, (2) epidermal growth factor (EGF) receptor-like cysteine-rich repeats, (3) YWTD domains, (4) a transmembrane 
domain, and (5) a cytoplasmic tail that harbors up to three NPXY motifs (Fig. 1) [2]. LRP1 consists of a large extracellular ligand-binding subunit $(515 \mathrm{kDa})$, transmembrane subunit, and short cytoplasmic tail ( $85 \mathrm{kDa})$ [10]. Structurally, LRP1 is made up of 31 class A cysteine-rich repeats and an extracellular domain comprised of 4 ligand-binding clusters (I-IV), which have $2,8,10$, and 11 complement-type repeats, respectively (Fig. 1) [11]. Domains II and IV mediate the binding of LRP1 to more than 40 ligands, including apolipoprotein, proteinases, proteinase-inhibitor complexes, bacterial toxins, viruses, the blood coagulation factor VIII, and various extracellular matrix proteins such as matrix metalloproteinases (MMPs) and urokinase-type

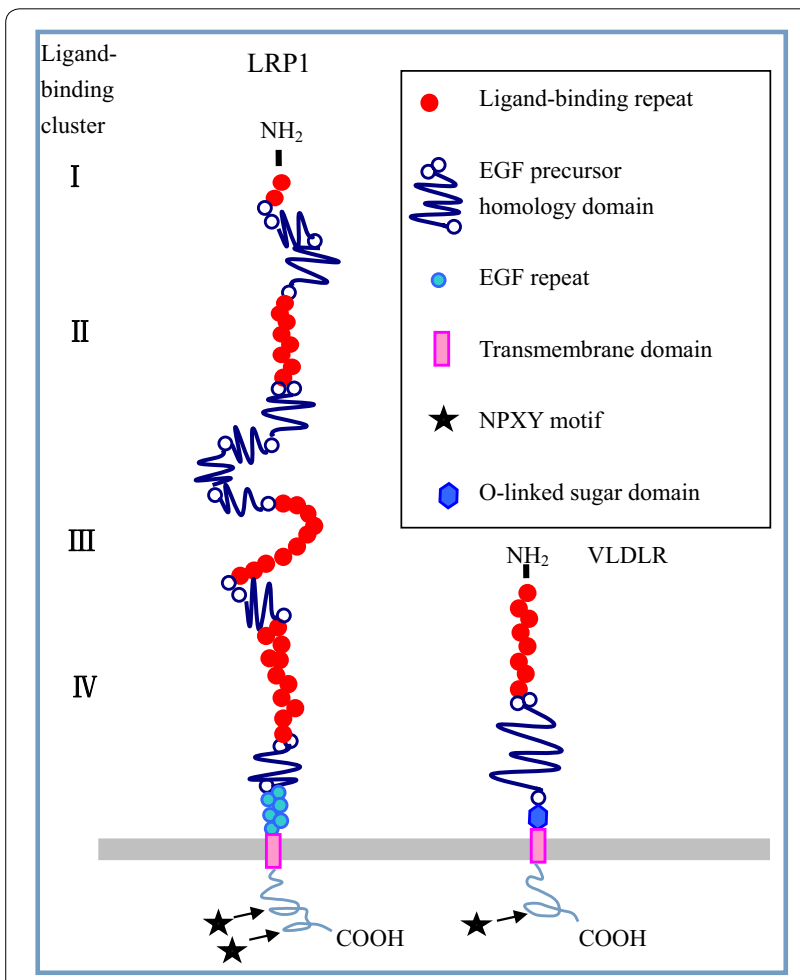

Fig. 1 Structures of low-density lipoprotein receptor-related protein 1 (LRP1) and very low-density lipoprotein receptor (VLDLR). This Figure is modified from Figure 1 of a review written by Herz et al. [2]. The large extracellular ligand-binding subunit of LRP1 consists of 4 different ligand-binging clusters that contain 2, 8, 10, and 11 cysteinerich ligand-binding repeats, respectively (red dots). Each cluster contains 1-4 epidermal growth factor (EGF) precursor homology domains, which include 2 cysteine-rich EGF repeats (blue circles) and 6 YWTD domains (blue lines). The cytoplasmic tail of LRP1 contains 2 NPXY motifs (marked with an asterisk). In addition, the cytoplasmic tail of LRP1 has 1 YXXL motif. Compared with LRP1, VLDLR is smaller. In addition, the O-linked sugar domain of VLDLR is enriched with serine and threonine residues (blue hexagon). Moreover, the cytoplasmic tail of VLDLR contains only 1 NPXY motif. Thus, the structure of LRP 1 determines the endocytosis of ligands plasminogen activator (uPA) [2, 12]. In contrast to LDLR, VLDLR, LRP2, and apoER2, the cytoplasmic tail of LRP1 contains 1 YXXL motif and 2 NPXY motifs (Fig. 1). The NPXY motifs function as the endocytosis signal for the LDLR family, and the YXXL motif serves as the dominant signal for rapid endocytosis of LRP1 $[13,14]$. Therefore, the primary function of LRP1 is to bind ligands via the extracellular ligand-binding domain and promote their internalization via its cytoplasmic tail. Furthermore, LRP1 delivers bound ligands to the endosomal/lysosomal compartment for release and then returns to the cell membrane for reuse [15]. In addition to the endocytosis of multiple ligands, more recent studies have reported that LRP1 interacts and activates a diverse set of signaling proteins. Recently, Mantuano et al. [5] reported that some proteins can bind to LRP1 and activate LRP1-dependent signaling molecules such as ERK1/2. Within this context, proteins such as tissuetype plasminogen activator (tPA), apoE, and activated $\alpha_{2}$-macroglobulin $\left(\alpha_{2} M\right)$ function as ligands of LRP1 [5]. In addition, substantial evidence indicates that LRP1 can activate various signaling pathways in response to particular ligands that bind to its extracellular domain by other mechanisms. For example, LRP1 binds to the platelet-derived growth factor (PDGF) receptor and forms a receptor-ligand complex that then activates the MAPK signaling pathway by driving endocytosis of the complex [4]. LRP1 has also been shown to activate signaling pathways by changing the levels of related receptors such as the uPA receptor (UPAR) [16]. Importantly, deregulation of the above signaling pathways has been implicated in tumor cell invasion and proliferation $[4,17]$. Therefore, it is likely that, in addition to clearing the extracellular environment, LRP1-mediated endocytosis of ligands contributes to the delivery of cancer-promoting signals in cells. Consequently, LRP1 is suggested to be involved in two major cell processes: endocytosis and modulation of signaling pathways, which play diverse biological roles in cancer cells. Taken together, the unique structure of LRP1 contributes to its multi-functionality.

\section{Association between LRP1 and cancer susceptibility}

The relationship between LRP1 and a variety of cancers has been reported. For example, Benes et al. [18] observed polymorphic alleles of C766 T in exon 3 of $L R P 1$ gene and a marked increase in the frequency of the $L R P 1 \mathrm{~T}$ allele in patients with breast cancer compared with control populations ( 0.21 versus $0.15, P=0.01963$ ), suggesting that the $\mathrm{T}$ allele of $\mathrm{C} 766 \mathrm{~T}$ in the $L R P 1$ gene may increase the risk of breast cancer. In addition, $L R P 1$ gene amplification has been found in astrocytoma, with 
$68 \%$ of high-grade astrocytomas (grade IV) exhibiting high expression of LRP1, compared with the lack of LRP1 expression observed in $91 \%$ of normal brain tissues [19]. Moreover, compared with low-grade astrocytoma, malignant glioma has been characterized with significantly higher levels of LRP1 mRNA and protein [20]. In addition, increasing levels of LRP1 expression is commonly observed in the majority of endometrial carcinomas, suggesting that LRP1 is involved in the formation of endometrial carcinoma [21]. Tumorigenesis is a multistep process that involves cancer cell proliferation, angiogenesis, invasion, and migration [22]. Therefore, it is likely that LRP1 is involved in modulating some of these processes.

\section{The role of LRP1 in regulating cancer cell invasion and migration} LRP1-mediated regulation of MMP expression promotes cancer cell migration and invasion

It is well known that MMPs, especially MMP-2 and MMP-9, are significantly involved in the invasion and metastasis of several types of human tumors [23, 24]. Recently, Song et al. [25] reported that LRP1 regulates the expression of MMP-2 and MMP-9, thereby promoting the migration and invasion of U87 human glioblastoma cells. In their study, knockdown of LRP1 using LRP1 small interfering RNA in the human glioblastoma cell line U87 led to a decrease in cell migration and invasion, which suggests that the expression level of LRP1 is associated with migration and invasion capabilities of these cancer cells. Using LRP1-deficient and LRP1overexpressing mouse embryonic fibroblasts cells, Song et al. [25] confirmed that LRP1-regulated MMP-2 and MMP-9 expression was involved in cell migration and invasion. In addition, they found that the level of phosphorylated ERK was decreased in LRP1-silenced cells, whereas other signaling pathways remained unchanged, suggesting that LRP1 regulates the expression of MMP-2 and MMP-9 via an ERK-dependent signaling pathway (Fig. 2) [25-27].

Other mechanisms have also been shown to contribute to LRP1-mediated MMP-dependent cancer cell invasion and migration. One study showed that the binding of the serine protease inhibitor protease nexin-1 (PN-1) to LRP1 stimulates MMP-9 expression, thereby promoting metastasis of breast cancer [28]. Similarly, $\alpha_{2} \mathrm{M}$ was shown to activate proMMP-2 via binding to LRP1 and promote the migration of human Müller glial cells [29]. However, $\alpha_{2} \mathrm{M}$ can also inhibit cell invasion through the clearance of pepsin from the extracellular environment [30, 31]. Thus, the role and mechanism of $\alpha_{2} \mathrm{M}$ in cell invasion and migration requires further investigation.

\section{LRP1-dependent activation of ERK and inhibition of JNK} promote cancer cell invasion

Another mechanism by which LRP1 promotes cancer cell invasion is via activation of ERK and inhibition of JNK signaling pathways, which are two major MAPK pathways (Fig. 2). One study found that the level of phosphorylated ERK-1/2 was selectively decreased in LRP1-silenced cancer cells, whereas the level of phosphorylated JNK-1/2/3 was increased in cells, suggesting that LRP1 may serve as an intracellular signaling regulator by regulating the ERK and JNK signaling pathways [17]. Furthermore, these results are consistent with those of previous studies performed in fibroblasts, Schwann cells, and other non-tumor cells $[32,33]$. In addition, the ERK signaling pathway has been shown to be activated during tumor cell invasion, and the invasion of JNK-overexpressing tumor cells is reduced, suggesting that ERK activation and JNK inhibition are required to promote tumor cell invasion [17]. Notably, the initial activation of the MAPK pathway is driven by the binding of LRP1 to PDGFR-bet in endosomes (Fig. 2) [4]. Future studies will be required to investigate the location of MAPK binding to LRP1.

\section{Role of the eHsp90-LRP1 signaling complex in tumor cell invasion and migration}

Gopal et al. [20] identified a novel extracellular heat shock protein 90 (eHsp90) that can bind to LRP1 and form an eHsp90-LRP1 signaling complex in glioblastoma multiforme (GBM) (Fig. 2). The eHsp90-LRP1 complex was shown to regulate activation of the receptor tyrosine kinase EphA2, which is frequently overexpressed in most GBMs and stimulates GBM cell motility and invasion following activation by AKT-dependent phosphorylation at S897 (p-EphA2S897) (Fig. 2). In addition, positive feedback signaling from eHsp90 further activates AKT and promotes recruitment of LRP1 to EphA2 (Fig. 2), thereby strengthening this signaling pathway and highlighting a novel mechanism of LRP1-mediated regulation of tumor cell migration and invasion. In addition, the aforementioned mechanism is augmented during cellular hypoxia, which subsequently enhances the eHsp90-LRP1 signaling axis due to increased expression of LRP1 and eHsp90 [20, 34]. Collectively, these studies provide novel insight into how eHsp90-LRP1 promotes tumor cell migration and invasion and may lead to new therapeutic approaches to suppress the aggressiveness of GBM.

\section{Controversial data concerning the role of LRP1 in cancer cell invasion and migration}

An increasing number of studies have confirmed that LRP1 promotes cancer cell invasion and migration. However, some investigations suggest that low expression of LRP1 can also promote tumor cell progression. uPA 


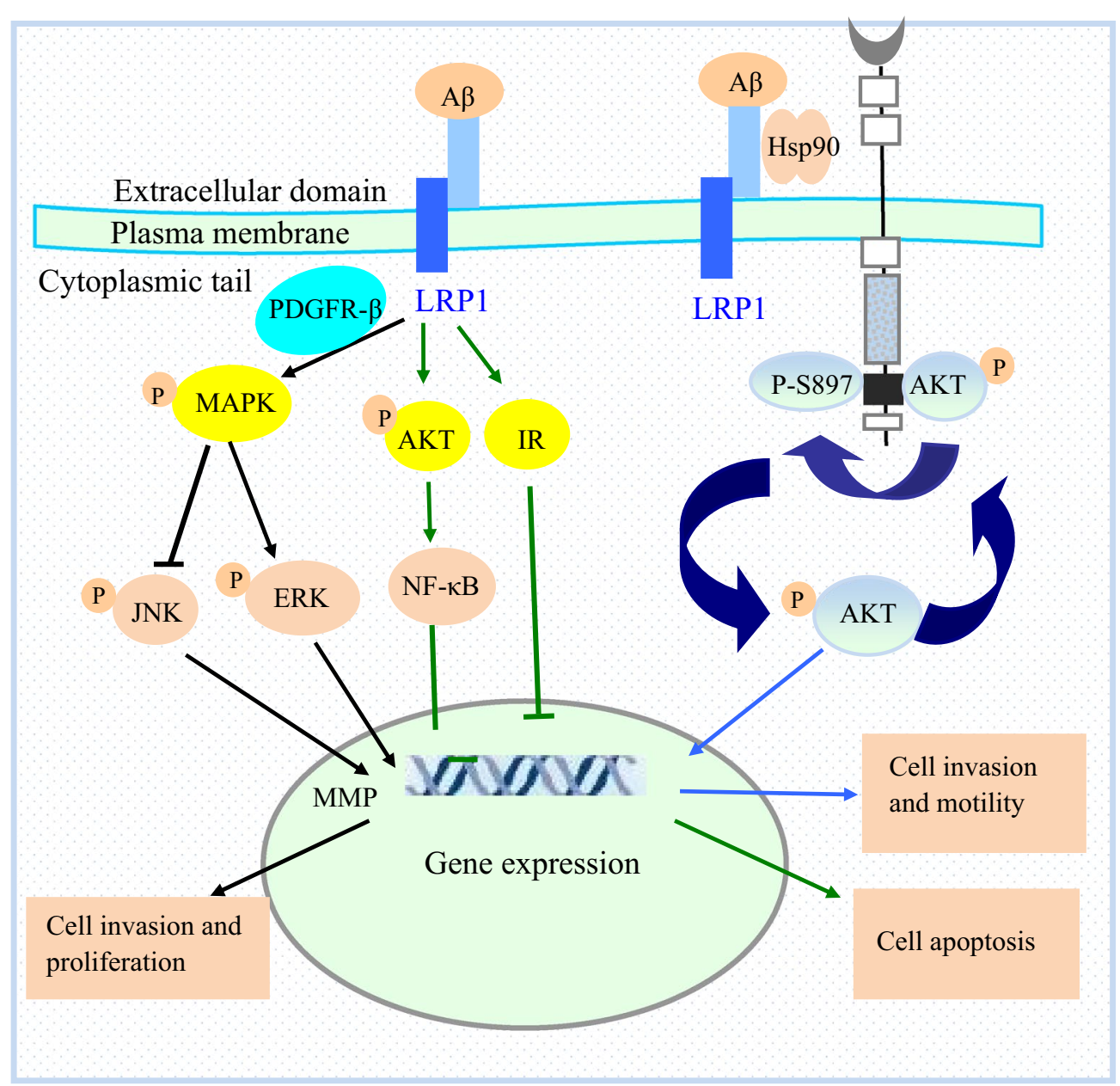

Fig. 2 Low-density LRP1-mediated cell signaling pathways. LRP1 regulates several signaling pathways in a phosphorylation-dependent manner. These pathways are involved in several processes of tumorigenesis and progression. Binding of LRP1 to platelet-derived growth factor (PDGF) receptor activates the mitogen-activated protein kinase (MAPK) signaling pathway, which subsequently activates the extracellular signal-regulated kinase (ERK) pathway and inhibits c-jun N-terminal kinase (JNK), resulting in cancer cell invasion and proliferation. In addition, ERK increases the transcriptional levels of matrix metalloproteinase (MMP)-2 and MMP-9, which drive cancer cell invasion. LRP1 activates the serine/threonine protein kinase (AKT) signaling pathway and insulin receptor (IR), which inhibits cancer cell apoptosis. LRP1 binds to the novel extracellular heat shock protein 90 (eHsp90) and forms an eHsp90-LRP1 signaling complex, which stimulates glioblastoma multiforme cell motility and invasion via AKT-dependent phosphorylation at 8897 (p-EphA2S897). The eHsp90 signaling regulates AKT activation and facilitates recruitment of LRP1 to the receptor tyrosine kinase EphA2

has been shown to be involved in tumor progression in a variety of cancers [35]. In human thyroid cancer cells, inhibiting LRP1 expression or increasing the level of uPA has been shown to enhance tumor cell invasion, specifically driving metastasis from lymph nodes and the lung [36]. Thus, low expression of LRP1 on the cell surface couples to increased expression and bioactivity of uPA and promotes tumor cell invasion [36]. Similarly, in fibroblasts, it has been reported that LRP1 inhibits cell migration by decreasing the cell surface abundance of uPA [16]. The mechanism of this function involves uPA binding to UPAR and activating plasminogen, which initiates a hydrolysis cascade of related proteins that drives degradation of the extracellular matrix. These processes facilitate tumor cell invasion through cell boundaries and hence, play a critical role in the migration of tumor and non-tumor cells [16]. Furthermore, a recent study has demonstrated that high expression of LRP1 is associated with a low metastatic potential of hepatocellular carcinoma (HCC) [37]. In addition, this study showed that inhibition of LRP1 increased the expression and bioactivity of MMP-9 in HCC cells. These results suggest a negative association between LRP1 and MMP-9 expression in HCC [37], which is in contrast to the observations made 
in U87 human glioblastoma cells [25]. Similar examples have also been found in other types of cancers, such as human endometrial carcinoma [38], breast and prostate cancers [39], lung cancer [40], and Wilm's tumors [41]. Therefore, these data suggest that LRP1 may have a dual role in cancer cell invasion and migration, which potentially depends on the different cell type and specific extracellular microenvironment.

\section{The role of LRP1 in regulating cancer cell apoptosis and proliferation}

Mounting evidence suggests that LRP1 plays an important role in cell proliferation and apoptosis. Fuentealba et al. [8] found that knockdown of LRP1 by short hairpin RNA in neurons significantly decreased the levels of Caspase-3 activation, AKT phosphorylation, IR signaling, and apoptosis. Caspase- 3 is a key enzyme in cell apoptosis, suggesting that LRP1 may also inhibit cancer cell apoptosis by regulating the activation of Caspase-3. Moreover, the important role of LRP1 in activating the AKT and IR signaling pathways has been confirmed in the forebrain of LRP1-ablated mice, which were characterized with decreased cell apoptosis, suggesting that LRP1 inhibits apoptosis by activating the IR and AKT signaling pathways (Fig. 2) [8]. Subsequent studies have revealed the mechanism by which LRP1 activates the AKT signaling pathway and that $\alpha_{2} \mathrm{M}$ can bind to LRP1 and activate AKT in Schwann cells [32]. However, the specific LRP1-mediated mechanism of activation remains unclear. In addition, one study reported that LRP1 is involved in PDGF-mediated activation of ERK phosphorylation, which can increase smooth muscle cell and fibroblast proliferation [4]. Thus, LRP1 can regulate cell apoptosis, proliferation, invasion, and migration.

\section{Unique role of monocyte- and macrophage-associated LRP1 in tumorigenesis and tumor progression}

Monocytes and macrophages promote cell invasion, metastasis, and angiogenesis in multiple cancers [42, 43]. In the initial step of tumor formation, macrophages create an inflammatory environment that promotes the growth of tumor cells. These growth-promoting effects on tumors are caused by the expression of multiple factors such as tumor necrosis factor- $\alpha$ and interleukin- 6 $[44,45]$. Moreover, macrophages produce EGF, which activates migration of tumor cells [46]. In addition, macrophages have been implicated in tumor angiogenesis. Accumulating studies reported that LRP1 in monocytes and macrophages plays a critical role in cancer progression by acting as a regulator of inflammation [45]. Staudt et al. [47] implanted PanO2 pancreatic carcinoma cells into mice that harbored LRP1-deficient myeloid cells and found that monocytes were strongly recruited to carcinoma cells. In addition, LRP1-deficient, bone marrow-derived macrophages expressed high levels of macrophage inflammatory protein-1 $\alpha /$ CCL3 [47]. These findings suggest a negative association between LRP1 expression and the recruitment of monocytes and macrophages. Moreover, tumor-associated, LRP1-deficient macrophages were characterized by the release of vascular endothelial growth factor (VEGF) into the tumor microenvironment [47]. The level of VEGF is closely associated with microvessel density and the malignant degree of tumors and is increasing in tumor tissues compared with non-tumor tissues [47], suggesting that VEGF may promote tumorigenesis by regulating LRP1 to enhance angiogenesis. Together, the roles of monocyteand macrophage-associated LRP1 in the initiation and progression of tumor suggest that targeted regulation of the inflammatory environment may prevent the occurrence of tumors.

\section{The multiple roles of $L R P 1 \mathrm{CpG}$ island methylation}

Accumulating evidence suggests that DNA methylation of $\mathrm{CpG}$ islands plays a critical role in the initiation and progression of multiple cancer types [48, 49]. The promoter region of $L R P 1$ is enriched with $\mathrm{CpG}$ islands that govern sensitivity of the $L R P 1$ gene to DNA methylation. Moreover, all constructs containing CpG sequences can increase transcriptional activity. Sonoda et al. [50] studied the methylation status of $L R P 1 \mathrm{CpG}$ islands in esophageal squamous cell carcinomas (ESCCs) and found that, when CpG was methylated completely, the transcriptional activity completely disappeared and the expression of LRP1 was silenced. Moreover, the methylation of LRP1 CpG islands was frequently observed in ESCC cells compared with control cells. Furthermore, when the expression of LRP1 was recovered, the growth of these ESCC cells was inhibited [50]. These data suggest that LRP1 CpG island methylation may be involved in tumor progression by controlling the expression of LRP1. In addition, methylation of $L R P 1 \mathrm{CpG}$ islands may represent a novel diagnostic marker for ESCC and other cancers.

\section{microRNA-205 (miR-205) and LRP1}

MicroRNAs are small noncoding RNAs that play a critical role in regulating post-transcriptional gene expression in various human cancers and are involved in driving tumorigenesis via regulation of metastasis, invasion, cell proliferation, and apoptosis [51]. Song et al. [52] examined U87 glioblastoma and SK-LU-1 lung adenocarcinoma cells, which express high levels of LRP1, and found that LRP1 expression and cell migration were both suppressed in cells transfected with miR-205. They also demonstrated that miR-205 suppressed LRP1 gene 
expression via inhibition of translation. Moreover, endocytosis of $\alpha_{2} \mathrm{M}$, a ligand of LRP1, was significantly suppressed in these cells [52]. Together, these data suggest that miR-205 down-regulates LRP1 expression and its endocytic function and leads to suppressed tumor cell migration and invasion [52]. Song et al. [52] also revealed the mechanism by which miR-205 causes LRP1 gene silencing. $m i R-205$ binds to its complementary sequences in the $3^{\prime}$ untranslated regions of $L R P 1$, which plays a role in regulating LRP1 expression. Consistent with this finding, Kajihara et al. [53] recently reported that $m i R-205$ down-regulated LRP1 expression and suppressed ERK phosphorylation, thereby inhibiting cell proliferation in dermatofibrosarcoma protuberans. Consequently, these results highlight a potential tumor suppressive function of $m i R-205$ via regulating the expression of LRP1.

\section{Novel LRP1-SNRNP25 fusion gene in osteosarcoma}

Recently, a novel fusion gene, LRP1-SNRNP25, was identified in human osteosarcoma by transcriptome sequencing, reverse transcription-polymerase chain reaction, and Sanger sequencing [54]. LRP1 and SNRNP25 are located on 12q and 16p, respectively. Sanger sequencing revealed that LRP1-SNRNP25 is formed by linking exon 8 of $L R P 1$ to exon 2 of SNRNP25. Fluorescence in situ hybridization not only confirmed that the fusion gene was resulted from interchromosomal rearrangement but also identified the amplification of the LRP1-SNRNP25 fusion gene. In addition, transfection of LRP1-SNRNP25 into SAOS-2 human osteosarcoma cells showed that the expression of LRP1-SNRNP25 promotes SAOS-2 cell migration and invasion $[54,55]$. Importantly, this novel finding may lead to a new therapeutic approach for osteosarcoma.

\section{Conclusions}

The ability of LRP1 to promote endocytosis and deliver cell signaling suggests that LRP1 may play multiple roles in tumorigenesis and tumor progression. In addition, LRP1 has dual effects on tumor cell invasion and migration, reinforcing that the tumor cell-specific activity of LRP1 must be studied in the future. Moreover, LRP1 can be regulated via methylation of $L R P 1 \mathrm{CpG}$ islands as well as $m i R-205$, which further complicates the role of LRP1 in cancer cells. LRP1 also serves as a regulator of monocytes and macrophages. Recently, the roles of the immune system in the initiation and progression of multiple cancers have received increasing attention. Thus, there are multiple opportunities to develop new LRP1-related diagnostic and therapeutic approaches for many types of cancers.

\section{Authors' contributions}

$J Y$ and PX designed and wrote the manuscript; ZL, ZR, JZ, and FS revised the manuscript; GW and KC co-designed, reviewed, and revised the manuscript. All authors read and approved the final manuscript.

\section{Author details}

${ }^{1}$ Department of Bone and Soft Tissue Tumor, Tianjin Medical University Cancer Institute and Hospital, Tianjin 30060, P. R. China. ${ }^{2}$ National Clinical Research Center for Cancer, Tianjin Medical University Cancer Institute and Hospital, Tianjin 30060, P. R. China. ${ }^{3}$ Department of Epidemiology and Biostatistics, Tianjin Medical University Cancer Institute and Hospital, Tianjin 30060, P. R. China.

\section{Acknowledgements}

This work was partly supported by the National Natural Science Foundation of China (81372872 to J. Yang, 81402215 to X. Du, and 81320108022 to K. Chen), funds from the University Cancer Foundation via the Sister Institution Network Fund at the Tianjin Medical University Cancer Institute and Hospital, Fudan University Shanghai Cancer Center, and University of Texas MD Anderson Cancer Center. This work was partly supported by the program for Innovative Research Team in University in China (IRT1076 to K. Chen).

\section{Competing interests}

The authors declare that they have no competing interests.

Received: 25 June 2015 Accepted: 12 October 2015

Published online: 06 January 2016

\section{References}

1. Herz J, Hamann U, Rogne S, Myklebost O, Gausepohl H, Stanley KK. Surface location and high affinity for calcium of a 500-kd liver membrane protein closely related to the LDL-receptor suggest a physiological role as lipoprotein receptor. EMBO J. 1988;7(13):4119-27.

2. Herz J, Strickland DK. LRP: a multifunctional scavenger and signaling receptor. J Clin Invest. 2001;108(6):779-84. doi:10.1172/jci13992.

3. Kang HS, Kim J, Lee HJ, Kwon BM, Lee DK, Hong SH. LRP1-dependent pepsin clearance induced by 2'-hydroxycinnamaldehyde attenuates breast cancer cell invasion. Int J Biochem Cell Biol. 2014;53:15-23. doi:10.1016/j.biocel.2014.04.021.

4. Muratoglu SC, Mikhailenko I, Newton C, Migliorini M, Strickland DK. Low density lipoprotein receptor-related protein 1 (LRP1) forms a signaling complex with platelet-derived growth factor receptor-beta in endosomes and regulates activation of the MAPK pathway. J Biol Chem. 2010;285(19):14308-17. doi:10.1074/jbc.M109.046672.

5. Mantuano E, Lam MS, Gonias SL. LRP1 assembles unique co-receptor systems to initiate cell signaling in response to tissue-type plasminogen activator and myelin-associated glycoprotein. J Biol Chem. 2013;288(47):34009-18. doi:10.1074/jbc.M113.509133.

6. Kasza A, Petersen HH, Heegaard CW, Oka K, Christensen A, Dubin A, et al. Specificity of serine proteinase/serpin complex binding to verylow-density lipoprotein receptor and alpha2-macroglobulin receptor/low-density-lipoprotein-receptor-related protein. Eur J Biochem. 1997;248(2):270-81.

7. Roura S, Cal R, Galvez-Monton C, Revuelta-Lopez E, Nasarre L, Badimon $\mathrm{L}$, et al. Inverse relationship between raft LRP1 localization and non-raft ERK1,2/MMP9 activation in idiopathic dilated cardiomyopathy: potential impact in ventricular remodeling. Int J Cardiol. 2014;176(3):805-14. doi:10.1016/j.ijcard.2014.07.270.

8. Fuentealba RA, Liu Q, Kanekiyo T, Zhang J, Bu G. Low density lipoprotein receptor-related protein 1 promotes anti-apoptotic signaling in neurons by activating Akt survival pathway. J Biol Chem. 2009;284(49):34045-53. doi:10.1074/jbc.M109.021030.

9. Schneider WJ, Nimpf J. LDL receptor relatives at the crossroad of endocytosis and signaling. Cell Mol Life Sci. 2003;60(5):892-903. doi:10.1007/ s00018-003-2183-Z.

10. Boucher P, Herz J. Signaling through LRP1: protection from atherosclerosis and beyond. Biochem Pharmacol. 2011;81(1):1-5. doi:10.1016/j. bcp.2010.09.018.

11. Neels JG, van Den Berg BM, Lookene A, Olivecrona G, Pannekoek H, van Zonneveld AJ. The second and fourth cluster of class A cysteine-rich repeats of the low density lipoprotein receptor-related protein share ligand-binding properties. J Biol Chem. 1999;274(44):31305-11.

12. Kanekiyo T, Bu G. The low-density lipoprotein receptor-related protein 1 and amyloid-beta clearance in Alzheimer's disease. Front Aging Neurosci. 2014;6:93. doi:10.3389/fnagi.2014.00093. 
13. Li Y, Marzolo MP, van Kerkhof P, Strous GJ, Bu G. The YXXL motif, but not the two NPXY motifs, serves as the dominant endocytosis signal for low density lipoprotein receptor-related protein. J Biol Chem. 2000;275(22):17187-94. doi:10.1074/jbc.M000490200.

14. Li Y, Lu W, Marzolo MP, Bu G. Differential functions of members of the low density lipoprotein receptor family suggested by their distinct endocytosis rates. J Biol Chem. 2001;276(21):18000-6. doi:10.1074/jbc M101589200.

15. von Arnim CA, Kinoshita A, Peltan ID, Tangredi MM, Herl L, Lee BM, et al. The low density lipoprotein receptor-related protein (LRP) is a novel beta-secretase (BACE1) substrate. J Biol Chem. 2005;280(18):17777-85. doi:10.1074/jbc.M414248200.

16. Weaver AM, Hussaini IM, Mazar A, Henkin J, Gonias SL. Embryonic fibroblasts that are genetically deficient in low density lipoprotein receptor-related protein demonstrate increased activity of the urokinase receptor system and accelerated migration on vitronectin. J Biol Chem. 1997;272(22):14372-9.

17. Langlois B, Perrot G, Schneider C, Henriet P, Emonard H, Martiny L, et al. LRP-1 promotes cancer cell invasion by supporting ERK and inhibiting JNK signaling pathways. PLoS One. 2010;5(7):e11584. doi:10.1371/journal. pone.0011584.

18. Benes P, Jurajda M, Zaloudik J, Izakovicova-Holla L, Vacha J. C766T lowdensity lipoprotein receptor-related protein 1 (LRP1) gene polymorphism and susceptibility to breast cancer. Breast Cancer Res. 2003;5(3):R77-81. doi:10.1186/bcr591.

19. Baum L, Dong ZY, Choy KW, Pang CP, Ng HK. Low density lipoprotein receptor related protein gene amplification and 766T polymorphism in astrocytomas. Neurosci Lett. 1998;256(1):5-8.

20. Gopal U, Bohonowych JE, Lema-Tome C, Liu A, Garrett-Mayer E, Wang $B$, et al. A novel extracellular Hsp90 mediated co-receptor function for LRP1 regulates EphA2 dependent glioblastoma cell invasion. PLoS One. 2011;6(3):e17649. doi:10.1371/journal.pone.0017649.

21. Catasus L, Llorente-Cortes V, Cuatrecasas M, Pons C, Espinosa I, Prat J. Low-density lipoprotein receptor-related protein 1 (LRP-1) is associated with highgrade, advanced stage and p53 and p16 alterations in endometrial carcinomas. Histopathology. 2011;59(3):567-71. doi:10.1111/j.1365-2559.2011.03942.x.

22. Liotta LA, Steeg PS, Stetler-Stevenson WG. Cancer metastasis and angiogenesis: an imbalance of positive and negative regulation. Cell. 1991;64(2):327-36.

23. Chambers AF, Matrisian LM. Changing views of the role of matrix metalloproteinases in metastasis. J Natl Cancer Inst. 1997;89(17):1260-70.

24. Wang M, Wang T, Liu S, Yoshida D, Teramoto A. The expression of matrix metalloproteinase-2 and -9 in human gliomas of different pathological grades. Brain Tumor Pathol. 2003;20(2):65-72.

25. Song H, Li Y, Lee J, Schwartz AL, Bu G. Low-density lipoprotein receptorrelated protein 1 promotes cancer cell migration and invasion by inducing the expression of matrix metalloproteinases 2 and 9. Cancer Res. 2009;69(3):879-86. doi:10.1158/0008-5472.can-08-3379.

26. Fink K, Boratynski J. The role of metalloproteinases in modification of extracellular matrix in invasive tumor growth, metastasis and angiogenesis. Postepy Hig Med Dosw (Online). 2012;66:609-28 [in Polish].

27. Hu K, Yang J, Tanaka S, Gonias SL, Mars WM, Liu Y. Tissue-type plasminogen activator acts as a cytokine that triggers intracellular signal transduction and induces matrix metalloproteinase-9 gene expression. J Biol Chem. 2006;281(4):2120-7. doi:10.1074/jbc.M504988200.

28. Fayard B, Bianchi F, Dey J, Moreno E, Djaffer S, Hynes NE, et al. The serine protease inhibitor protease nexin-1 controls mammary cancer metastasis through LRP-1-mediated MMP-9 expression. Cancer Res. 2009;69(14):5690-8. doi:10.1158/0008-5472.can-08-4573.

29. Barcelona PF, Jaldin-Fincati JR, Sanchez MC, Chiabrando GA. Activated alpha2-macroglobulin induces Muller glial cell migration by regulating MT1-MMP activity through LRP1. Faseb J. 2013;27(8):3181-97. doi:10.1096/fi.12-221598.

30. Athauda SB, Nishigai M, Arakawa H, Ikai A, Ukai M, Takahashi K. Inhibition of human pepsin and gastricsin by alpha2-macroglobulin. J Enzyme Inhib Med Chem. 2003;18(3):219-24

31. Johnston N, Yan JC, Hoekzema CR, Samuels TL, Stoner GD, Blumin JH, et al. Pepsin promotes proliferation of laryngeal and pharyngeal epithelial cells. Laryngoscope. 2012;122(6):1317-25. doi:10.1002/lary.23307.
32. Mantuano E, Inoue G, Li X, Takahashi K, Gaultier A, Gonias SL, et al. The hemopexin domain of matrix metalloproteinase-9 activates cell signaling and promotes migration of schwann cells by binding to low-density lipoprotein receptor-related protein. J Neurosci. 2008;28(45):11571-82. doi:10.1523/jneurosci.3053-08.2008.

33. Takayama Y, Takahashi H, Mizumachi K, Takezawa T. Low density lipoprotein receptor-related protein (LRP) is required for lactoferrin-enhanced collagen gel contractile activity of human fibroblasts. J Biol Chem. 2003;278(24):22112-8. doi:10.1074/jbc.M300894200.

34. Montel V, Gaultier A, Lester RD, Campana WM, Gonias SL. The lowdensity lipoprotein receptor-related protein regulates cancer cell survival and metastasis development. Cancer Res. 2007;67(20):9817-24. doi:10.1158/0008-5472.can-07-0683.

35. Sidenius N, Blasi F. The urokinase plasminogen activator system in cancer: recent advances and implication for prognosis and therapy. Cancer Metastasis Rev. 2003;22(2-3):205-22

36. Sid B, Dedieu S, Delorme N, Sartelet H, Rath GM, Bellon G, et al. Human thyroid carcinoma cell invasion is controlled by the low density lipoprotein receptor-related protein-mediated clearance of urokinase plasminogen activator. Int J Biochem Cell Biol. 2006;38(10):1729-40. doi:10.1016/j. biocel.2006.04.005.

37. Huang XY, Shi GM, Devbhandari RP, Ke AW, Wang Y, Wang XY, et al. Low level of low-density lipoprotein receptor-related protein 1 predicts an unfavorable prognosis of hepatocellular carcinoma after curative resection. PLoS One. 2012;7(3):e32775. doi:10.1371/journal.pone.0032775.

38. Foca C, Moses EK, Quinn MA, Rice GE. Differential expression of the alpha(2)-macroglobulin receptor and the receptor associated protein in normal human endometrium and endometrial carcinoma. Mol Hum Reprod. 2000;6(10):921-7.

39. Kancha RK, Stearns ME, Hussain MM. Decreased expression of the low density lipoprotein receptor-related protein/alpha 2-macroglobulin receptor in invasive cell clones derived from human prostate and breast tumor cells. Oncol Res. 1994;6(8):365-72.

40. Meng H, Chen G, Zhang X, Wang Z, Thomas DG, Giordano TJ, et al. Stromal LRP1 in lung adenocarcinoma predicts clinical outcome. Clin Cancer Res. 2011;17(8):2426-33. doi:10.1158/1078-0432.ccr-10-2385.

41. Desrosiers RR, Rivard ME, Grundy PE, Annabi B. Decrease in LDL receptorrelated protein expression and function correlates with advanced stages of Wilms tumors. Pediatr Blood Cancer. 2006;46(1):40-9. doi:10.1002/ pbc.20566.

42. Laoui D, Movahedi K, Van Overmeire E, Van den Bossche J, Schouppe E, Mommer C, et al. Tumor-associated macrophages in breast cancer: distinct subsets, distinct functions. Int J Dev Biol. 2011;55(7-9):861-7. doi:10.1387/ijdb.113371dl.

43. Zhang BC, Gao J, Wang J, Rao ZG, Wang BC, Gao JF. Tumor-associated macrophages infiltration is associated with peritumoral lymphangiogenesis and poor prognosis in lung adenocarcinoma. Med Oncol. 2011;28(4):1447-52. doi:10.1007/s12032-010-9638-5.

44. Naugler WE, Sakurai T, Kim S, Maeda S, Kim K, Elsharkawy AM, et al. Gender disparity in liver cancer due to sex differences in MyD88dependent IL-6 production. Science. 2007;317(5834):121-4. doi:10.1126/ science.1140485.

45. Qian BZ, Pollard JW. Macrophage diversity enhances tumor progression and metastasis. Cell. 2010;141(1):39-51. doi:10.1016/j.cell.2010.03.014.

46. Wyckoff J, Wang W, Lin EY, Wang Y, Pixley F, Stanley ER, et al. A paracrine loop between tumor cells and macrophages is required for tumor cell migration in mammary tumors. Cancer Res. 2004;64(19):7022-9. doi:10.1158/0008-5472.can-04-1449.

47. Staudt ND, Jo M, Hu J, Bristow JM, Pizzo DP, Gaultier A, et al. Myeloid cell receptor $L R P 1 / C D 91$ regulates monocyte recruitment and angiogenesis in tumors. Cancer Res. 2013;73(13):3902-12. doi:10.1158/0008-5472. can-12-4233.

48. Qu Y, Dang S, Hou P. Gene methylation in gastric cancer. Clin Chim Acta. 2013;424:53-65. doi:10.1016/j.cca.2013.05.002

49. Ehrlich M, Jiang G, Fiala E, Dome JS, Yu MC, Long TI, et al. Hypomethylation and hypermethylation of DNA in Wilms tumors. Oncogene. 2002;21(43):6694-702. doi:10.1038/sj.onc.1205890.

50. Sonoda I, Imoto I, Inoue J, Shibata T, Shimada Y, Chin K, et al. Frequent silencing of low density lipoprotein receptor-related protein 1B (LRP1B) expression by genetic and epigenetic mechanisms in 
esophageal squamous cell carcinoma. Cancer Res. 2004;64(11):3741-7. doi:10.1158/0008-5472.can-04-0172.

51. Esquela-Kerscher A, Slack FJ. Oncomirs - microRNAs with a role in cancer. Nat Rev Cancer. 2006;6(4):259-69. doi:10.1038/nrc1840.

52. Song H, Bu G. MicroRNA-205 inhibits tumor cell migration through down-regulating the expression of the LDL receptor-related protein 1. Biochem Biophys Res Commun. 2009;388(2):400-5. doi:10.1016/j. bbrc.2009.08.020.

53. Kajihara I, Jinnin M, Harada M, Makino K, Honda N, Makino T, et al. miR205 down-regulation promotes proliferation of dermatofibrosarcoma protuberans tumor cells by regulating LRP-1 and ERK phosphorylation. Arch Dermatol Res. 2014;306(4):367-74. doi:10.1007/s00403-014-1452-z.
54. Yang J, Annala M, Ji P, Wang G, Zheng H, Codgell D, et al. Recurrent LRP1SNRNP25 and KCNMB4-CCND3 fusion genes promote tumor cell motility in human osteosarcoma. J Hematol Oncol. 2014;7(1):76. doi:10.1186/ s13045-014-0076-2.

55. Yang $\mathrm{J}$. Investigation of osteosarcoma genomics and its impact on targeted therapy: an international collaboration to conquer human osteosarcoma. Chin J Cancer. 2014;33(12):575-80. doi:10.5732/cjc.014.10209.
Submit your next manuscript to BioMed Central and we will help you at every step:

- We accept pre-submission inquiries

- Our selector tool helps you to find the most relevant journal

- We provide round the clock customer support

- Convenient online submission

- Thorough peer review

- Inclusion in PubMed and all major indexing services

- Maximum visibility for your research

Submit your manuscript at www.biomedcentral.com/submit
Biomed Central 